\title{
Carotenoid Profiles in Pigment-Protein Complexes of Rhodospirillum rubrum
}

\author{
Rosmarie Ursula Schwerzmann and Reinhard Bachofen
}

\author{
Institut für Pflanzenbiologie der Universität Zürich, Zollikerstrasse 107, CH-8008 Zürich, Switzerland
}

\begin{abstract}
Young cells of Rhodospirillum rubrum contain a set of carotenoids from lycopene to spirilloxanthin. During growth, intermediates are almost completely converted to spirilloxanthin. The ratio of the different carotenoid precursors vs. spirilloxanthin found in material of a certain age is the same in cells, chromatophores, light-harvesting complexes and reaction centers. Independent of the carotenoid composition and the age of the cells, the same detergent treatment can be used for isolation of pigment-protein complexes. Light-harvesting complexes of young cells containing mainly precursors of spirilloxanthin, as well as those of old cells in which spirilloxanthin dominates, both have their absorption maximum at $880 \mathrm{~nm}$. It is thus assumed that all carotenoids of the spirilloxanthin series interact with bacteriochlorophyll a similarly to spirilloxanthin itself.

From these results it is concluded that the micro-environment of these membrane-complexes is not influenced by the type of carotenoid present and that the assembly of the pigment-protein complexes in a growing membrane takes place before carotenoid biosynthesis has lead to the final product.
\end{abstract}

Key words: Carotenogenesis - Diphenylamine - Light-harvesting complex — Reaction center Rhodospirillum rubrum $\mathrm{S} 1$ - Spirilloxanthin precursor.

Phototrophic bacteria appear in various colours from yellow brown to deep red, due to the different absorption properties of carotenoids which act as the main coloured cell constituents. In such cells these lipid soluble com-

\footnotetext{
Abbreviations: anhydrorhodovibrin, 1-methoxy-3,4-didehydro-1,2-dihydro- $\psi, \psi$-carotene; chloroxanthin, 1,2, $7^{\prime}, 8^{\prime}$ tetrahydro- $\psi, \psi$-caroten-1-ol; 3,4-dehydrorhodopin, 3,4-didehydro-1,2-dihydro- $\psi, \psi$-caroten-1-ol; $11^{\prime}, 12^{\prime}$-dihydrospheroidene, 1-methoxy-3,4-didehydro-1,2,7', $8^{\prime}, 11^{\prime}, 12^{\prime}$-hexahydro- $\psi, \psi$-carotene; lycopene, $\psi, \psi$-carotene; neurosporene, 7,8-dihydro- $\psi, \psi$-carotene; $\mathrm{OH}$-spheroidene, $1^{\prime}$-methoxy-3',4'-didehydro-1,2,7,8,1',2'hexahydro- $\psi, \psi$-caroten-1-ol; OH-spirilloxanthin, 1'-methoxy$3,4,3^{\prime}, 4^{\prime}$-tetradehydro-1,2,1',2'-tetrahydro- $\psi, \psi$-caroten-1-ol; phytoene, $7,8,11,12,7^{\prime}, 8^{\prime}, 11^{\prime}, 12^{\prime}$-octahydro- $\psi, \psi$-carotene; phytofluene, $7,8,11,12,7^{\prime}, 8^{\prime}$-hexahydro- $\psi, \psi$-carotene; rhodopin, 1,2-dihydro$\psi, \psi$-caroten-1-ol; rhodovibrin, 1'-methoxy-3',4'-didehydro-1,2,1',2'tetrahydro- $\psi, \psi$-caroten-1-ol; spheroidene, 1-methoxy-3,4-didehydro-1,2,7', $8^{\prime}$-tetrahydro- $\psi, \psi$-carotene; spirilloxanthin, $1,1^{\prime}$-dimethoxy-3,4,3',4'-tetradehydro-1,2,1',2'-tetrahydro- $\psi, \psi$-carotene; $7,8,11,12$-tetrahydrolycopene, 7,8,11,12-tetrahydro- $\psi, \psi$-carotene;

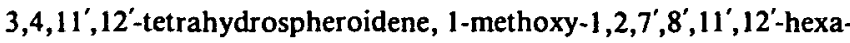
hydro- $\psi, \psi$-carotene; DPA, diphenylamine; LHC, light-harvesting pigment-protein complex; $\mathrm{RC}$, reaction center.
}

pounds are bound to integral membrane proteins, the LHC and the RC. In the natural habitats of phototrophic bacteria carotenoids serve as accessory pigments in trapping light energy, furthermore they are important in protecting the photosynthetic apparatus from photooxidation (Cogdell 1985, Siefermann-Harms 1985). Carotenoids fround in phototrophic bacteria are mainly acyclic and often contain hydroxyl-, methyl- and keto-groups. Several pathways of carotenoid biosynthesis in phototrophic bacteria have been described (Schmidt 1978). The spirilloxanthin family which is present in many of the species investigated, was first demonstrated in Rhodospirillum rubrum (Jensen et al. 1958, Jensen et al. 1961). The conversion of lycopene to spirilloxanthin involves hydroxylation, desaturation and methylation at both sides of the polyene chain. Initially one side of the molecule seems to be modified by these reactions followed by the other one. Lycopene, rhodopin, anhydrorhodovibrin, rhodovibrin as well as the end product spirilloxanthin are all found in relatively large quantities in growing cells of $R$. rubrum S-1. In comparison in late exponential and stationary phase cultures, more than 95\% of total carotenoid is usually found as spirilloxanthin. In contrast, several other strains of $R$. rubrum as well as other species of phototrophic bacteria which belong to 
the spirilloxanthin group contain much less of the end product spirilloxanthin, even in old cultures. In these cases, any of the spirilloxanthin precursors may be the dominating carotenoid species (Schmidt 1978).

In the elucidation of the biosynthesis of carotenoids, diphenylamine has been used as an inhibitor of in vivo carotenoid biosynthesis. Intermediates more saturated than lycopene were accumulated in the cell, providing an insight into some of the early desaturating reactions (Davies 1970a).

In the work cited above, whole cells have been investigated and therefore, no information on the intracellular localization of these biochemical transformations is available.

During the last few years, several methods have been developed to isolate light-harvesting complexes (Cogdell et al. 1982, Picorel et al. 1983) as well as reaction centers (Van der Rest and Gingras 1974, Snozzi and Bachofen 1979) from $R$. rubrum and other phototrophic bacteria (Drews 1985). A stoichiometry of one molecule of spirilloxanthin to two molecules of bacteriochlorophyll a, one spirilloxanthin to four bacteriochlorophyll a and two bacteriopheophytin a is usually given for light-harvesting complexes and reaction centers, respectively (Evans et al. 1988, Picorel et al. 1983, Van der Rest and Gingras 1974). In all these investigations the membrane complexes have been isolated from old cultures in the stationary growth phase and spirilloxanthin has been reported comprising up to $100 \%$ of the carotenoid fraction.

In a linear biosynthetic chain, visible accumulation of intermediate products is only expected if the enzyme activities of successive steps are unbalanced or if the enzymes are activated in a sequence within the chain. When different species of carotenoids occur during the life time of an organism it is necessary to determine whether the carotenoids finally found incorporated in the membrane are inserted in their final form, e.g. as spirilloxanthin, or whether the biosynthetic modifications leading to the end product spirilloxanthin occur when the molecule is already protein-bound, either to the light-harvesting complexes or the reaction centers.

In this study light-harvesting complexes and reaction centers have been isolated from cultures of different age and analysed for their carotenoid composition.

\section{Materials and Methods}

Rhodospirillum rubrum S-1 was grown anaerobically in the light in the medium of Ormerod et al. (1961) in $1,000 \mathrm{ml}$ Pyrex bottles. Inoculation from a stock culture was carried out to give an initial absorbance of 0.065 at $880 \mathrm{~nm}$. Incubation was at $30^{\circ} \mathrm{C}$ under constant stirring for homogeneous illumination at a fluence rate of $20 \mathrm{~W} / \mathrm{m}^{2}$ for 24 to $144 \mathrm{~h}$ as indicated in the results. To inhibit nor- mal carotenoid synthesis, cells were grown as described by Jensen et al. (1958) at a DPA concentration of $70 \mu \mathrm{M}$. Chromatophores were isolated as described by Snozzi and Bachofen (1979). Light-harvesting complexes were prepared according to the method a) of Picorel et al. (1983). Reaction centers were purified from the first supernatant of light-harvesting complex preparation according to Snozzi and Bachofen (1979).

Pigments were extracted from chromatophores at $0^{\circ} \mathrm{C}$ by a ten-fold volume of acetone/methanol $7: 2$ at a concentration giving a final absorption at $771 \mathrm{~nm}$ of $0.1-0.2$. After centrifugation at $2,000 \times g$, the spectrum of the supernatant was recorded on a Uvikon 810 spectrophotometer. The pigments were quantified using the equations and extinction coefficients of Van der Rest and Gingras (1974) (see Table 1), which correct for overlapping bands in the absorption spectra of carotenoids, bacteriochlorophyll a and bacteriopheophytin a. For samples containing a variety of carotenoids the extinction coefficient of spirilloxanthin was used for all species.

From isolated pigment-protein complexes, pigments were extracted as follows: $2 \mathrm{ml}$ of methanol, $2 \mathrm{ml}$ of dichloromethane and $1 \mathrm{ml}$ of a $1 \%$ sodium chloride solution were added to $0.8 \mathrm{ml}$ of aqueous solution and the mixture vortexed vigorously. After centrifugation at $2,000 \times g$, the water/methanol phase and the interphase were discarded. The dichloromethane phase was dried under vacuo and the residue dissolved in acetone/methanol $7: 2$. Spectra were recorded as described above.

For separation of the pigments by HPLC, samples were extracted by the dichloromethane/methanol method. The dichloromethane phase was either injected directly or, in the presence of lauryldimethylamine oxide (LDAO), the solvent was evaporated and the pigments dissolved in acetone before injection. This procedure causes LDAO to precipitate on the walls of the test tube. The absorption of samples at $771 \mathrm{~nm}$ was in the range 20 to 40. Samples were loaded by a $20 \mu \mathrm{l}$ loop on a Spherisorb S5 ODS 2 column $(250 \times 4.6 \mathrm{~mm})$ and eluted isocratically with methanol/tetrahydrofurane $92: 8$. For scanning spectra of individual peaks, a peak was captured within the cuvette of the Uvikon 722 LC spectrophotometer by a stop flow valve and recorded on a Spectra Physics SP 4100 computing integrator. Identification of carotenoids was done by comparison of the absorption spectra with literature values (Jensen et al. 1958, Davies 1970a, Davies 1970b) and by their chromatographic behaviour. Neurosporene and lycopene were verified by co-chromatography of a pigment extract of Rhodobacter sphaeroides GA or authentic lycopene (Roth, Karlsruhe, F. R. G.), respectively. Carotenoids of the spirilloxanthin series were monitored at $475 \mathrm{~nm}$. Quantification as in Figure 4 was carried out as follows: individual peaks were collected and absorption spectra in methanol/tetrahydrofurane $92: 8$ recorded on a 
Uvikon 810 spectrophotometer. As reliable extinction coefficients are missing, the recommended value for unknown carotenoids was used $\left(\varepsilon^{1 \%}=2,500\right)$ (Jensen and Jensen 1971, Lichtenthaler 1987). For $475 \mathrm{~nm}$, the following extinction coefficients were calculated: lycopene and rhodopin: 2,500; anhydrorhodovibrin and rhodovibrin: 2,150; spirilloxanthin: 1,850 . The peak areas at $475 \mathrm{~nm}$ were corrected with these values and the amount of individual carotenoids in relation to the total carotenoid was calculated.

\section{Results}

Absorption spectra of growing cultures of Rhodospirillum rubrum show important changes in the shape of the carotenoid absorption bands. In $24 \mathrm{~h}$ old cultures, the carotenoid absorption band has only one absorption maximum at $500 \mathrm{~nm}$ and is of relatively undefined structure. During the subsequent growth of the culture, the carotenoid absorption band develops more fine-structure and in $120 \mathrm{~h}$ old cells, the carotenoid region shows the conventional three maxima with the main absorption at $510 \mathrm{~nm}$.

A similar sequence is seen in isolated pigment-protein complexes. Figure 1 shows absorption spectra of lightharvesting complexes after dialysis isolated from cells of different ages. The initial maximum at approx. $500 \mathrm{~nm}$ in light-harvesting complexes of $24 \mathrm{~h}$ old cells shifts to $510 \mathrm{~nm}$ and higher in $120 \mathrm{~h}$ cells. At the same time, a sharpening and an increase of the carotenoid absorption band occurs relative to the $880 \mathrm{~nm}$ band. The same age-dependent redshift in the carotenoid bands is also observed in reaction centers; but it is less pronounced because of the overlap of the bacteriopheophytin a band with the carotenoid absorption at around $545 \mathrm{~nm}$. It must be noted that the absorption band of bacteriochlorophyll at $880 \mathrm{~nm}$ and its Soret band do not shift in wavelength during growth and are not influenced by the type of carotenoid bound to the pigmentprotein complexes.

For the determination of carotenoid to bacteriochloro-

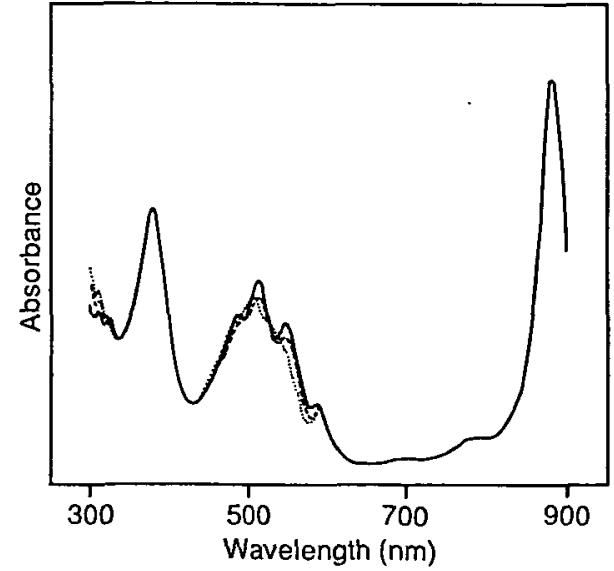

Fig. 1 Absorption spectra of LHC isolated from cells of $R$. rubrum $\mathrm{Sl}$ grown anaerobically in the light for $24 \mathrm{~h}(\cdots), 72 \mathrm{~h}$ $(---), 120 \mathrm{~h}(-)$.

phyll a ratios during growth, chromatophores were extracted with the widely used mixture of acetone/methanol $7: 2$. Since the carotenoid composition and thus the absorption spectra in the carotenoid region change during growth, total concentration of carotenoids was determined using three different wavelengths for calculation. For the wavelengths and corresponding extinction coefficients of bacteriochlorophyll and carotenoids see Table 1. The small overlap in absorption of bacteriopheophytin was neglected. The resulting ratios of carotenoids to bacteriochlorophyll are given in Fig. 2. Within experimental error, the carotenoid to bacteriochlorophyll a ratio remains constant during the whole growth period. However in Fig. $2 b$ and $2 c$, where the carotenoid extinctions at $525 \mathrm{~nm}$ or at the actual absorption maximum are used for calculation, a slight increase of the carotenoid to bacteriochlorophyll a ratio seems to occur. Evaluating the optimal detection wavelength for HPLC separation of the pigments, $475 \mathrm{~nm}$ was favoured for the following reasons: (i) $525 \mathrm{~nm}$ is to far away from the mean main absorption

Table 1 Millimolar extinction coefficients $\left(\mathrm{mM}^{-1} \cdot \mathrm{cm}^{-1}\right)$ for bacteriochlorophyll a and carotenoids in acetone/methanol

\begin{tabular}{lcccr}
\hline Pigment & \multicolumn{3}{c}{ Wavelength } \\
\cline { 2 - 5 } & $771 \mathrm{~nm}^{a}$ & $525 \mathrm{~mm}^{a}$ & $475 \mathrm{~mm}^{a}$ & Main abs. max. of car. ${ }^{b}$ \\
\hline Bacteriochlorophyll a & 65.3 & 1.5 & 1.9 & - \\
Carotenoids & 0.0 & 101.4 & 94.0 & 149.0
\end{tabular}

\footnotetext{
${ }^{a}$ From van der Rest and Gingras (1974).

${ }^{b}$ Millimolar extinction coefficient for carotenoids is based on $\varepsilon^{1 \%} \approx 2,500$ for unknown carotenoids (Jensen and Jensen 1971 ) and the molecular weight for spirilloxanthin of 596 .
} 


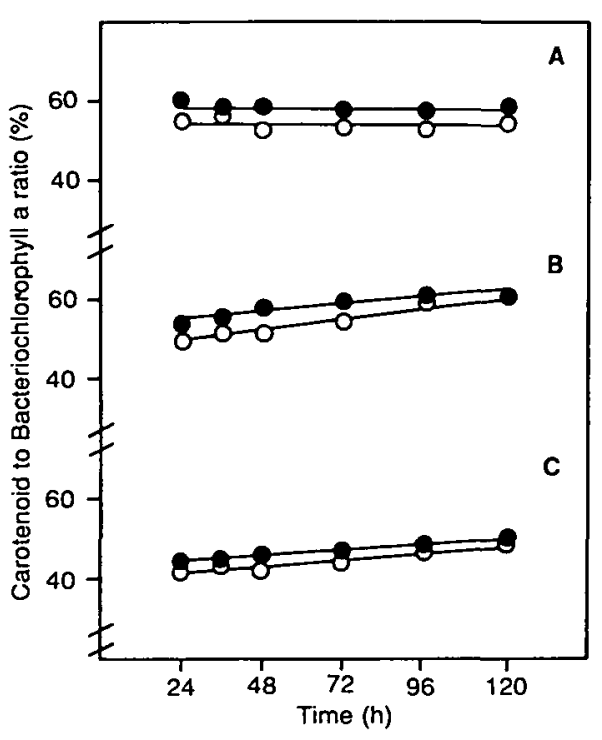

Fig. 2A, B, C Ratio of carotenoids to bacteriochlorophyll a during photo-heterotrophic growth. Spectra were recorded in acetone/methanol. Chromatophore-pigments were either extracted by acetone/methanol (•) or dichloromethane/methanol (O). Detection wavelengths for carotenoids: A $475 \mathrm{~nm}$. B $525 \mathrm{~nm}$. C main absorption maximum of carotenoids. (Max. relative standard errors: $2.1 \%$ for acetone/methanol method; 7.9\% for dichloromethane/methanol method).

maximum of the investigated carotenoids (Table 2 , peaks 2-6), thus especially lycopene and rhodopin could hardly be detected, (ii) as the position of the main absorption max- imum of a pigment extract depends on the carotenoid composition, the detection wavelength should be adjusted to each sample individually. Comparison of different chromatograms would present considerable difficulties.

For our experiments, the acetone/methanol procedure described had several disadvantages: it is not applicable to LDAO-containing samples and the resulting pigment concentration is too low for subsequent HPLC separation. The use of the second procedure, whereby the pigments were extracted by dichloromethane/methanol, overcame these problems. Carotenoid to bacteriochlorophyll ratios determined accordingly result in somewhat lower ratios of carotenoids to bacteriochlorophyll (Fig. 2). However, the slopes of the time curves are similar to the ones of the corresponding acetone/methanol-extracts, we take this as an indication that the two methods are equivalent with respect to the extraction of the different carotenoid compounds found. This holds true also for extracts obtained from chromatophores and from light-harvesting complexes.

Figure 3 shows the separation of the pigments of the light-harvesting complex by HPLC. Absorption spectra of each peak were recorded during chromatography. The measured main and long wavelength absorption maxima are listed in Table 2 and compared with literature values. Together with the expected chromatographic behaviour, individual carotenoids were identified as indicated in Table 2. Clearly the chromatographic peaks of rhodovibrin, spirilloxanthin and anhydrorhodovibrin do not show Gaussian distribution but are followed by distinct shoulders. These compounds absorb at slightly lower wavelengths than the preceeding main carotenoid and furthermore

Table 2 Position of main and long wavelength absorption maxima [nm] of carotenoid compounds from Figure 3 (Peaks 2-6) and Figure 5 (Peaks 4, 7-13)

\begin{tabular}{|c|c|c|c|c|}
\hline \multirow{2}{*}{ Peak } & \multirow{2}{*}{$\begin{array}{l}\text { This work (methanol/ } \\
\text { tetrahydrofurane } 92: 8 \text { ) }\end{array}$} & \multirow{2}{*}{ Identification $^{a}$} & \multicolumn{2}{|c|}{ Literature values (petroleum ether) } \\
\hline & & & Davies $1970 \mathrm{~b}$ & Jensen 1958 \\
\hline 2 & $479 / 512$ & rhodovibrin & & $482 / 515$ \\
\hline 3 & $469 / 499$ & rhodopin & & $474 / 506$ \\
\hline 4 & $489 / 521$ & spirilloxanthin & $489 / 524$ & $491 / 524$ \\
\hline 5 & $479 / 513$ & anhydrorhodovibrin & $482 / 515$ & $482 / 514$ \\
\hline 6 & $468 / 498$ & lycopene & $473 / 505$ & $474 / 506$ \\
\hline 7 & $453 / 483$ & OH-spheroidene & $453 / 484$ & \\
\hline 8 & $438 / 468$ & chloroxanthin & $439 / 467$ & \\
\hline 9 & $455 / 483$ & spheroidene & $453 / 484$ & \\
\hline 10 & $392 / 418 / 438$ & $\begin{array}{l}3,4,11^{\prime}, 12^{\prime} \text {-tetrahydrospheroidene } \\
\text { and } 11^{\prime}, 12^{\prime} \text {-dihydrospheroidene }\end{array}$ & $\begin{array}{l}394 / 418 \\
414 / 440\end{array}$ & \\
\hline 11 & $438 / 468$ & neurosporene & $440 / 467$ & $438 / 468$ \\
\hline 12 & $345 / 365 / 392 / 418$ & $\begin{array}{l}\text { phytofluene and } \\
7,8,11,12 \text {-tetrahydrolycopene }\end{array}$ & $347 / 367$ & $\begin{array}{l}347 / 367 \\
394 / 418\end{array}$ \\
\hline 13 & $285 /$ shoulder at 295 & phytoene & $285 / 296$ & $284 / 296$ \\
\hline
\end{tabular}

\footnotetext{
The differences in peak wavelengths determined in this paper compared to the literature values are due to solvent effects (Vetter et al.
} 1971). 
show a strong absorption band at 370 in the cases of rhodovibrin and anhydrorhodovibrin or 382 in the case of spirilloxanthin in the so-called cis-region. They are the cisisomers of the preceeding all-trans carotenoids. Extinction coefficients of cis-isomers are much lower than the corresponding value of the trans-compounds (Vetter et al. 1971). Since on a reverse phase column cis-/trans-isomers are not completely separated, each peak consists of a mixture of at least two isomeric forms. In this situation, to quantify the carotenoid pattern in cells, chromatophores and pigment-protein complexes, one is left with the extinction coefficients of the pigments described (see Materials and Methods).

In light-harvesting complexes and reaction centers of $24 \mathrm{~h}$ old cells, the main carotenoids were rhodovibrin, spirilloxanthin and anhydrorhodovibrin, with smaller

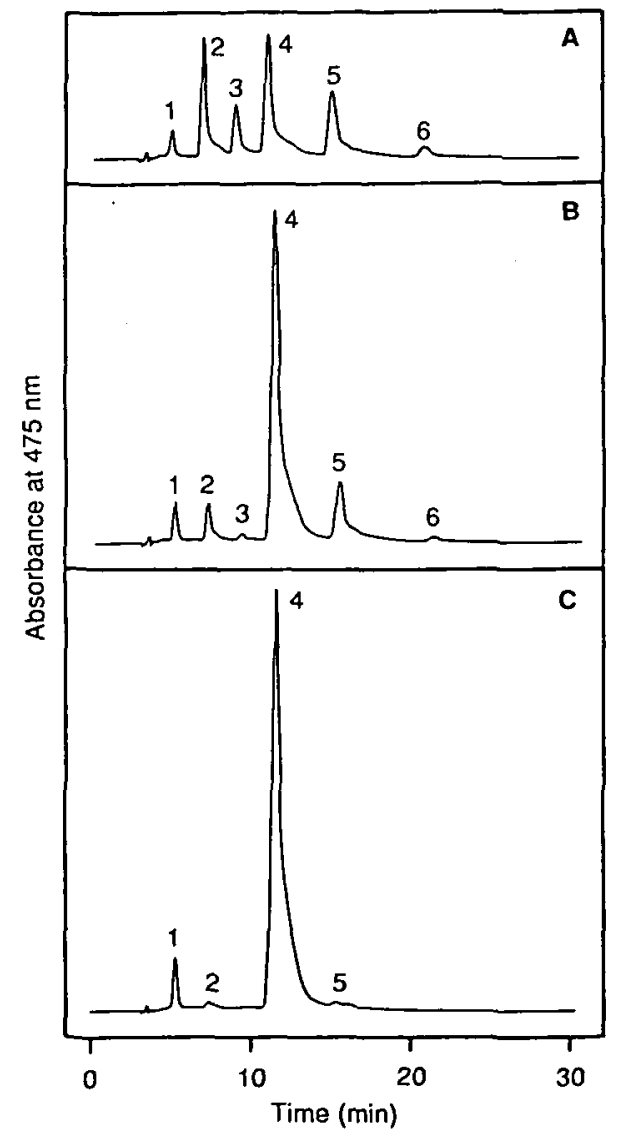

Fig. 3A, B, C HPLC-separation of total pigments extracted from LHC (absorbance of extracts at $771 \mathrm{~nm}$ was 30). Age of cells used for LHC-isolation: A $24 \mathrm{~h}$. B $72 \mathrm{~h}$. C $120 \mathrm{~h}$. Separation-conditions: Spherisorb S5 ODS 2 column $(250 \times 4.6 \mathrm{~mm})$; methanol/tetrahydrofurane $92: 8 ; 1 \mathrm{ml} / \mathrm{min}$; detection wavelength $475 \mathrm{~nm}$. Identification of peaks: (1) bacteriochlorophyll a, (2) rhodovibrin, (3) rhodopin, (4) spirilloxanthin, (5) anhydrorhodovibrin, (6) lycopene. amounts of rhodopin and lycopene. In all cases, lycopene was the earliest carotenoid found in samples deriving from cells grown under anaerobic conditions in the light, it never exceeded $3 \%$ of total carotenoid. During growth of the culture, rhodovibrin, rhodopin, anhydrorhodovibrin and lycopene disappeared, while spirilloxanthin increased. In pigment-protein complexes of cells from $120 \mathrm{~h}$ cultures spirilloxanthin accounted for over $95 \%$ of total carotenoids. Other possible intermediates such as 3,4-dehydrorhodopin and $\mathrm{OH}$-spirilloxanthin were never detected.

With all preparations a similar accumulation of the main precursors rhodovibrin and anhydrorhodovibrin in young material and an increase of spirilloxanthin in older material was observed (Fig. 4). This indicates that the isolation procedures for chromatophore membranes, the use of detergents for the preparation of light-harvesting complexes and reaction centers do not preferentially extract or disintegrate complexes of a certain pigment composition. Furthermore neither ion exchange chromatography nor dialysis, procedures common in the preparation of light-harvesting complexes and reaction centers, had an influence on the carotenoid profile. In $24 \mathrm{~h}$ cells the relative amount of spirilloxanthin is highest in reaction centers. This is due to the following reasons: (i) Bacteriopheophytin, which was not separated from samples prior to chromatography, elutes together with cis-spirilloxanthin. As the absorption of bacteriopheophytin is minimal at the detection wavelength of $475 \mathrm{~nm}$, its influence was neglected in the calculation of the spirilloxanthin concentration. (ii)

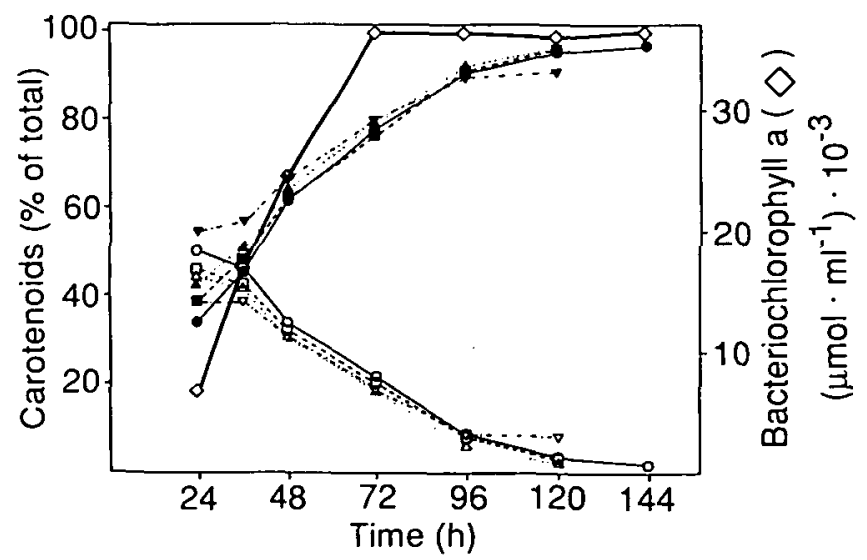

Fig. 4 Determination and quantification of carotenoids by HPLC. Relative concentration of spirilloxanthin (closed symbols) and its quantitatively main precursors (anhydrorhodovibrin +rhodovibrin) (open symbols) in cells $(\bullet, 0)$, chromatophores $(\square$, $\square)$, light-harvesting complexes $(\Delta, \Delta)$, and reaction centers $(\nabla, \nabla)$. (Max. relative standard errors: $5 \%$ ). Calculation of carotenoid amounts was done as described in Materials and Methods. Bacteriochlorophyll a concentration after acetone/methanol $7: 2$ extraction of cultures of $R$. rubrum $(0)\left(\varepsilon=65.3 \mu \mathrm{mol}^{-1} \cdot \mathrm{ml}\right)$. 
Rhodopin (not shown in Fig. 4) reached 10-15\% in cells, chromatophores and light-harvesting complexes after $24 \mathrm{~h}$, while in reaction centers it only accounted for $5 \%$.

The total bacteriochlorophyll a content increases during the first $72 \mathrm{~h}$ and remains constant afterwards. As the carotenoid to bacteriochlorophyll a ratio does not change during the whole growth period (Fig. 2) it is concluded that carotenoid net biosynthesis stops after $72 \mathrm{~h}$. At that time, only about $80 \%$ of total carotenoid are found as spirilloxanthin. The remaining $20 \%$ of total carotenoid are found to be lycopene, rhodopin, anhydrorhodovibrin and rhodovibrin (Fig. 3b). During the following stationary growth phase, these precursors are converted to spirilloxanthin in the absence of net biosynthesis of carotenoids.

Light-harvesting complexes from cells grown under conditions where normal carotenogenesis is inhibited by addition of $70 \mu \mathrm{M}$ diphenylamine accumulated large amounts of the precursor phytoene with minor amounts of phytofluene, 7,8,11,12-tetrahydrolycopene and neurosporene (Fig. 5 and Table 2). With the exception of spirilloxanthin itself, no carotenoid of the normal spirilloxanthin series (see Fig. 3a) was found under carotenogenesis inhibiting conditions. The remaining chromatographic peaks $(7,8,9$ and 10$)$ contain coloured carotenoids with the electronic absorption properties listed in Table 2 . Comparing these data with literature values suggested the identification given in Table 2. These compounds are formed as side products by hydroxylation, desaturation and

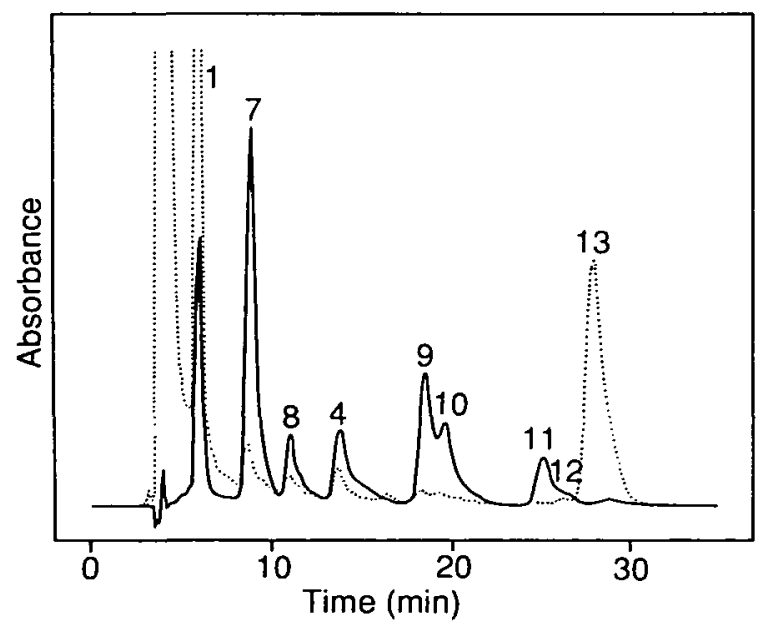

Fig. 5 HPLC-separation of pigments extracted from LHC isolated from cultures grown in the presence of $70 \mu \mathrm{M}$ DPA. Detection wavelengths $440 \mathrm{~nm}(-)$ and $300 \mathrm{~nm}(\cdots)$. Other conditions as in Figure 3. Identification of peaks: (1) bacteriochlorophyll a, (4) spirilloxanthin, (7) OH-spheroidene, (8) chloroxanthin, (9) spheroidene, (10) $3,4,11^{\prime}, 12^{\prime}$,-tetrahydrospheroidene $+11^{\prime}, 12^{\prime}$-dihydrospheroidene, (11) neurosporene, (12) phytofluene $+7,8,11,12$-tetrahydrolycopene, (13) phytoene. methylation of carotenoids more saturated than lycopene (Davies 1970b, Malhotra et al. 1970, Davies and Than 1974). Over such side-pathways, still relatively large amounts of spirilloxanthin are formed. With respect to the near-infrared absorption band and the carotenoid profiles in cells, chromatophores, light-harvesting complexes and reaction centers, the same holds true for diphenylamine-inhibited cultures as for cells grown under normal conditions.

\section{Discussion}

In cells of old cultures of Rhodospirillum rubrum spirilloxanthin is the main caroterioid, while in cells of young cultures various precursors like lycopene, rhodopin, anhydrorhodovibrin and rhodovibrin are dominant (Van Niel et al. 1956, Jensen et al. 1958). In preparations of light-harvesting complexes and reaction centers the same general carotenoid profile and the same sequence of appearance in the biosynthetic pathway was found as in whole cells and chromatophores of the same culture. The earliest species of carotenoid found in pigment-protein complexes of cells grown under normal conditions was lycopene. The decrease in relative as well as absolute amounts of precursors cannot be due to the dilution of precursors by spirilloxanthin but is caused by the transformation of precursors to spirilloxanthin during growth.

In contrast in the presence of diphenylamine, more saturated carotenoids such as phytoene, phytofluene, $7,8,11,12$-tetrahydrolycopene and neurosporene are accumulated in the pigment-protein complexes. Under normal growth conditions, phytoene is not detected, probably because it is rapidly desaturated to lycopene, which is the first tetraterpene compound found in young cells and in their pigment-protein complexes. From a physiological point of view, a rapid desaturation of phytoene is important, as only carotenoids with at least nine conjugated double bonds (neurosporene) are capable of protecting the photosynthetic apparatus from photodestruction (Krinsky 1978). During exponential growth phase, modifying reactions to form spirilloxanthin occur at a decreased rate compared to bacteriochlorophyll synthesis. At the end of exponential growth phase of a culture (as measured by bacteriochlorophyll a content), about $80 \%$ of carotenoids are spirilloxanthin. The remaining ones are transformed during the following stationary phase to reach finally over $95 \%$ of spirilloxanthin after $120 \mathrm{~h}$. Within the boundaries of experimental error, the total carotenoid to bacteriochlorophyll ratio is $1: 2$ (as recently shown by Evans et al. 1988) and remains constant during the whole growth period.

The fact that light-harvesting complexes and reaction centers with different carotenoid compositions are isolated during the cell cycle by the same detergent treatment indicates that the molecular environment in the vicinity of 
these membrane complexes does not depend on the nature of the carotenoid molecules bound to the complex. The wavelength of the near-infrared absorption maximum of the light-harvesting complex around $873 \mathrm{~nm}$ in the carotenoidless mutant $R$. rubrum G-9 is shifted to $880 \mathrm{~nm}$ in wildtype cells due to the interaction of bacteriochlorophyll a with carotenoids (Miller et al. 1987). Since in lightharvesting complexes of normally grown $R$. rubrum S-1 as well as in the presence of diphenylamine, the position of the absorption maximum stays at $880 \mathrm{~nm}$ during the whole growth period, it follows that all precursors of spirilloxanthin are bound to the light-harvesting and reaction center polypeptides in the same way and at the same site as spirilloxanthin. Similar assumptions have been made from reconstitution experiments with different carotenoids into the B800-850 complex of Rhodobacter sphaeroides $\mathbf{R}$ 26 (Davidson and Cogdell 1981) or into reaction centers of R. rubrum G-9 (Boucher et al. 1977).

Our results demonstrate that assembling of the pigments with the polypeptides of light-harvesting complexes and reaction centers takes place when an early precursor of spirilloxanthin, probably phytoene, becomes available in the membrane. However transformation of this precursor to the final carotenoid requires desaturation, hydroxylation and methylation reactions at both ends of the polyene chain. Although in Rhodospirillaceae no enzyme involved in carotenoid formation has so far been characterized, these processes seem to be catalyzed by enzymes integrally bound to the membrane (see Bramley 1985 for review) and these modifications take place after the precursor has been attached to the polypeptides. However it is still an open question (i) whether the enzymes can act on carotenoids while they are bound to the pigment-protein complexes, (ii) whether a direct exchange of carotenoids between pigment-protein complexes to modifying enzymes takes place or (iii) whether carotenoids bound to pigmentprotein complexes move into the lipid phase where they are handled by the appropriate enzymes. The last case seems unlikely, as upon LDAO-solubilization of chromatophores, such loosely bound carotenoids might easily be removed from the pigment-protein complexes. This would question the constant carotenoid to bacteriochlorophyll a ratios found. To decide between (i) and (ii), detailed enzymologic investigations are a prerequisite.

We thank Dr. P. Rüedi (Institute for Organic Chemistry, University of Zurich) for helpful discussions concerning separation and identification of carotenoids. This work was supported by the Swiss National Foundation for Scientific Research (grant 3.243-0.85).

\section{References}

Boucher, F., van der Rest, M. and Gingras, G. (1977) Structure and function of carotenoids in the photoreaction center of Rhodospirillum rubrum. Biochim. Biophys. Acta 461: 339357.

Bramley, P. M. (1985) The in vitro biosynthesis of carotenoids. Adv. Lipid Res. 21: 243-279.

Cogdell, R. J. (1985) Carotenoids in photosynthesis. Pure Appl. Chem. 57: 723-728.

Cogdell, R. J., Lindsay, J. G., Valentine, J. and Durant, I. (1982) A further characterisation of the B890 light-harvesting pigment-protein complex from Rhodospirillum rubrum strain S1. FEBS Lett. 150: 151-154.

Davidson, E. and Cogdell, R. J. (1981) Reconstitution of carotenoids into the light-harvesting pigment-protein complex from the carotenoidless mutant of Rhodopseudomonas sphaeroides R26. Biochim. Biophys. Acta 635: 295-303.

Davies, B. H. (1970a) A novel sequence for phytoene dehydrogenation in Rhodospirillum rubrum. Biochem. J. 116: 93-99.

Davies, B. H. (1970b) Alternative pathways of spirilloxanthin biosynthesis in Rhodospirillum rubrum. Biochem. J. 116: 101-110.

Davies, B. H. and Than, A. (1974) Monohydroxycarotenoids from diphenylamine-inhibited cultures of Rhodospirillum rubrum. Phytochemistry 13: 209-219.

Drews, G. (1985) Structure and functional organization of lightharvesting complexes and photochemical reaction centers in membranes of phototrophic bacteria. Microbiol. Rev. 49: 5970.

Evans, M. B., Cogdell, R. J. and Britton, G. (1988) Determination of the bacteriochlorophyll : carotenoid ratios of the $\mathrm{B} 890$ antenna complex of Rhodospirillum rubrum and the B800-850 complex of Rhodobacter sphaeroides. Biochim. Biophys. Acta 935: 292-298.

Jensen, S. L., Cohen-Bazire, G., Nakayama, T. O. M. and Stanier, R. Y. (1958) The path of carotenoid synthesis in a photosynthetic bacterium. Biochim. Biophys. Acta 29: 477-498.

Jensen, S. L., Cohen-Bazire, G. and Stanier, R. Y. (1961) Biosynthesis of carotenoids in purple bacteria: a re-evaluation based on considerations of chemical structure. Nature (London) 192: 1168-1172.

Jensen, S. L. and Jensen, A. (1971) Quantitative determination of carotenoids in photosynthetic tissues. Methods Enzymol. 23: 586-602.

Krinsky, N. I. (1978) Non-photosynthetic functions of carotenoids. Phil. Trans. R. Soc. Lond. B 284: 581-590.

Lichtenthaler, H.K. (1987) Chlorophylls and carotenoids: pigments of photosynthetic biomembranes. Methods Enzymol. 148: 350-382.

Malhotra, H. C., Britton, G. and Goodwin, T. W. (1970) The mono- and dimethoxy-carotenoids of diphenylamine-inhibited cultures of Rhodospirillum rubrum. Phytochemistry 9: 23692375.

Miller, J. F., Hinchigeri, S. B., Parkes-Loach, P. S., Callahan, 
P. M., Sprinkle, J. R., Riccobono, J. R. and Loach, P. A. (1987) Isolation and characterization of a subunit form of the light-harvesting complex of Rhodospirillum rubrum. Biochemisry 26: 5055-5062.

Ormerod, J. G., Ormerod, K. S. and Gest, H. (1961) Lightdependent utilization of organic compounds and photoproduction of molecular hydrogen by photosynthetic bacteria; relationships with nitrogen metabolism. Arch. Biochem. Biophys. 94: 449-463.

Picorel, R., Bélanger, G. and Gingras, G. (1983) Antenna holochrome B880 of Rhodospirillum rubrum S1. Pigment, phospholipid, and polypeptide composition. Biochemistry 22: 249l-2497.

Schmidt, K. (1978) Biosynthesis of carotenoids. In The photosynthetic Bacteria. Edited by Clayton, R. K. and Sistrom, W. R. pp. 729-750. Plenum Press, New York.
Siefermann-Harms, D. (1985) Carotenoids in photosynthesis. I. Location in photosynthetic membranes and light-harvesting function. Biochim. Biophys. Acta 811: 325-355.

Snozzi, M. and Bachofen, R. (1979) Characterisation of reaction centers and their phospholipids from Rhodospirillum rubrum. Biochim. Biophys. Acta 546: 236-247.

Van der Rest, M. and Gingras, G. (1974) The pigment complement of the photosynthetic reaction center isolated from Rhodospirillum rubrum. J. Biol. Chem. 249: 6446-6453.

Van Niel, C. B., Goodwin, T. W. and Sissins, M. E. (1956) Studies in carotenogenesis: 21 . The nature of the changes in carotenoid synthesis in Rhodospirillum rubrum during growth. Biochem. J. 63: 408-412.

Vetter, W., Englert, G., Rigassi, N. and Schwieter, U. (1971) Spectroscopic methods. In Carotenoids. Edited by Isler, $\mathrm{O}$. pp. 189-266. Birkhäuser, Basel.

(Received October 26, 1988; Accepted March 6, 1989) 